\title{
Performance of Multi-antenna Array Assisted MC DS-CDMA Using Downlink Preprocessing Based on Singular Value Decomposition
}

\author{
Chong $\mathrm{Xu}$, Bin Hu, Lie-Liang Yang and Lajos Hanzo \\ School of ECS., Univ. of Southampton, SO17 1BJ, UK. \\ Tel: +44-23-80-593 125, Fax: +44-23-80-593045 \\ Email: \{cx05r, lly, lh\}@ecs.soton.ac.uk, http://www-mobile.ecs.soton.ac.uk
}

\begin{abstract}
In this contribution we propose and investigate a transmitter preprocessing scheme for downlink transmission in the multicarrier direct-sequence code-division multiple-access (MC DS-CDMA) systems using multiple base-station antenna arrays and each antenna array using multiple array elements. The transmitter preprocessing scheme is derived based on the singular value decomposition (SVD) approach. Our transmitter preprocessing design motives to support a high number of users by the MC DS-CDMA, to achieve the highest achievable diversity gain and to result in low-complexity detection for the remote mobilestations (MS's). In this contribution the characteristics of the MC DSCDMA using the proposed transmitter preprocessing is discussed and the bit-error-rate (BER) performance is investigated, when assuming that each subcarrier experiences flat Rayleigh fading. Our study and simulation results show that, for a SVD-assisted MC DS-CDMA system using $M$ transmit antenna arrays and the time ( $\mathrm{T}$ )-domain spreading sequences of length $N_{e}$, the number of users supportable can be as high as $M N_{e}$ contributed by both the T-domain and S-domain. Furthermore, the SVD-assisted MC DS-CDMA system is capable of supporting such a high number of users with near single-user BER performance achieved by a corresponding MC DS-CDMA system supporting only one downlink user.
\end{abstract}

\section{INTRODUCTION}

In CDMA wireless communications the MC DS-CDMA is recognized as a high-flexibility multiple-access scheme, which is capable of providing a higher number of degrees-of-freedom for system design and online reconfiguration, in comparison with both the singlecarrier DS-CDMA and frequency $(\mathrm{F})$-domain spread multicarrier CDMA (MC-CDMA) without using T-domain spreading [1]-[3]. In [4], [5] the authors have proposed and investigated a MC DSCDMA system, which employs multiple base-station (BS) antenna arrays in order to achieve the receive diversity [4] for the uplink or the transmit diversity [5] for the downlink, both in the S-domain. Furthermore, as shown in [4], [5], when the receiver or transmitter beamforming is employed, the proposed MC DS-CDMA system is capable of suppressing the interfering signals having their directionof-arrivals (DoAs) that are different from that of the desired signal. To be more specific, in [5] the performance of the multi-antenna array assisted MC DS-CDMA has been investigated, when the BS transmitter preprocesses the transmitted signals using a hybrid of steered beamforming and space-time spreading (SSTS), which makes use of the knowledge about the DoAs of all the downlink users, but not the knowledge about the channel impulse responses (CIRs) of the downlink channels. In this case the downlink users experience severe multiuser interference (MUI), which may only be mitigated at the remote MS's with the aid of advanced multiuser detection (MUD) schemes [6].

Recently, transmitter preprocessing techniques have received wide attention and research in wireless communications, as seen, e.g., in [7]-[12]. It has been shown that, with the aid of transmitter preprocessing, in cellular wireless communications systems using time-division duplex (TDD), the downlink diversity may be achieved and the downlink MUI may be mitigated by carrying out the required signal processing at the BS. Consequently, power-efficient MS's may be implemented with low-complexity algorithms. Therefore, in this contribution we investigate the performance of the MC DSCDMA system considered in [4], [5], when assuming the TDD-based cellular communications and that the BS transmitter can exploit the knowledge about both the DoAs and the CIRs in the context of all the downlink users. Specifically, we assume that the BS transmitter preprocesses the downlink transmitted signals using a transmitter preprocessing scheme derived based on the SVD principles. The transmitter preprocessing scheme is designed so that the MC DSCDMA is capable of supporting a high number of users and achieving the highest achievable diversity gain with low-complexity MS detectors. Our analysis and simulation results show that the performance of the MC DS-CDMA can be significantly enhanced, when our SVD-assisted transmitter preprocessing is employed. Specifically, when using $M$ transmit antenna arrays and the T-domain spreading sequences of length $N_{e}$, the MC DS-CDMA system is capable of supporting upto $M N_{e}$ users, while achieving the near single-user BER performance. Furthermore, good trade-off between the number of users supported and the achievable diversity gain can be reached, as detailed in our forthcoming discourse.

\section{SYSTEM DESCRIPTION}

In this section we consider the transmitter and receiver models of our MC DS-CDMA system using base-station (BS) smart antennas, which employs downlink transmitter preprocessing based on the principles of SVD.

\section{A. Transmitter Model}

Fig. 1 shows the transmitter schematic block diagram of the downlink MC DS-CDMA system, where the BS employs $M$ transmit antenna arrays, each of which consists of $L$ array elements. We assume that the $M$ antenna arrays are set sufficiently apart from each other, in order that their CIRs are independent. By contrast, the $L$ array elements of an antenna array are arranged close to each other, in order to achieve the beamforming gain, which can be configurated for enhancing the signals in certain direction, while simultaneously degrading the signals in the other directions. As shown in Fig. 1 the MC DS-CDMA has a total of $U V$ number of subcarriers. We assume that each of the subcarrier signals experiences flat fading, even though 
the overall MC DS-CDMA signal experiences frequency-selective fading.

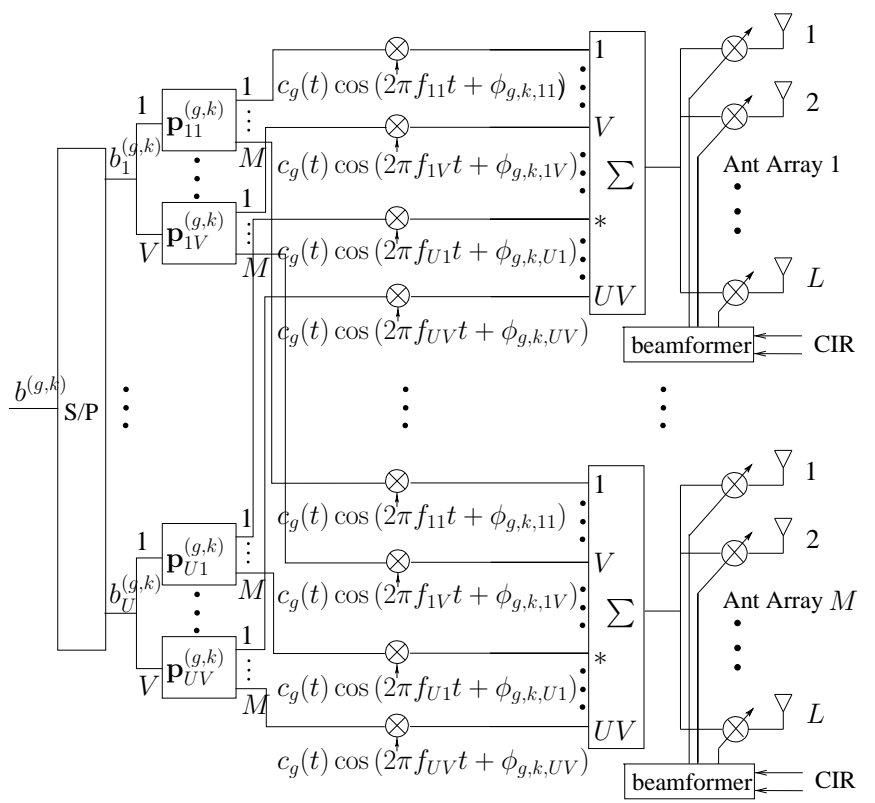

Fig. 1: Downlink transmitter schematic diagram for the MC DSCDMA employing $M$ transmit antennas, each of which has $L$ array elements, when BS transmitter preprocessing is employed.

We assume that the MC DS-CDMA supports $\mathcal{K}=K G$ downlink users, which is divided into $G$ groups and each group contains $K$ users. The $K$ users in a given group, say group $g$, share a common T-domain spreading sequence expressed as $\mathbf{c}_{g}=$ $\left[c_{g}[0], \cdots, c_{g}\left[N_{e}-1\right]\right]^{T}$ of length $N_{e}$. Furthermore, it is assumed that $\mathbf{c}_{g}$ is orthogonal to $\mathbf{c}_{q}$, when $g \neq q$. Let, after the 1-to- $U$ serialto-parallel (S/P) conversion in Fig. 1, the data bits to be transmitted to the $k$ th downlink user in the $g$ th group be expressed as

$$
\mathbf{b}^{(g, k)}=\left[b_{1}^{(g, k)}, b_{2}^{(g, k)}, \ldots, b_{U}^{(g, k)}\right]^{T}
$$

where one bit is transmitted by invoking $V$ subcarriers. Therefore, the total number of subcarriers in the MC DS-CDMA system is $U V$. Specifically, the subcarriers invoked for transmitting the $u$ th, $u=$ $1, \ldots, U$, bit of the $k$ th user in the $g$ th group are $\left\{f_{u 1}, f_{u 2}, \ldots, f_{u V}\right\}$.

As shown in Fig. 1, in the context of the uvth subcarrier, the data bit $b_{u}^{(g, k)}$ is first preprocessed by a $M$-length vector $\mathbf{p}_{u v}^{(g, k)}$, yielding $M$ outputs corresponding to the $M$ transmit antennas. After the preprocessing the signals are then spread using the Tdomain spreading code $c_{g}(t)=\sum_{n=0}^{N_{e}-1} c_{g}[n] P_{T_{c}}\left(t-n T_{c}\right)$, where $P_{T_{c}}(t)$ represents the typical rectangular chip waveform of width $T_{c}, T_{c}$ represents the chip-duration and, furthermore, $N_{e}=T_{s} / T_{c}$ represents the spreading factor, $T_{s}=U T_{b}$ is the symbol-duration, while $T_{b}$ is the bit-duration. As shown in Fig. 1, following the T-domain spreading, the signals are carrier modulated and, finally, transmitted by the $M$ antenna arrays, where the array elements may be weighted correspondingly based on certain optimization criterion with the aid of the knowledge about the CIRs of the downlink channels.

Based on the above description, it can be shown that the baseband equivalent signals transmitted to the $K$ users in the $g$ th group can be expressed as

$$
\begin{aligned}
\mathbf{s}^{(g)}(t)= & \sqrt{\frac{2 P}{V L} \sum_{k=1}^{K} \sum_{u=1}^{U} \sum_{v=1}^{V} \mathbf{W}^{(g, k)} \mathbf{p}_{u v}^{(g, k)} b_{u}^{(g, k)}} \\
& \times c_{g}(t) \exp \left(2 \pi f_{u v} t+\phi_{u v}^{(g, k)}\right) \\
g & =1,2, \ldots, G
\end{aligned}
$$

where $P$ represents the transmitted power to each of the $K$ users, $V L$ is a power normalization factor, where $V$ is due to the $V$ subcarriers conveying the same data bit, while $L$ is due to the number of array elements. Furthermore, in (2) $\mathbf{W}^{(g, k)}$ is the $(M L \times M)$-dimensional transmitter beamforming matrix for the $M$ antenna arrays, which can be expressed as

$$
\mathbf{W}^{(g, k)}=\left[\begin{array}{cccc}
\mathbf{w}_{1}^{(g, k)} & \mathbf{0} & \cdots & \mathbf{0} \\
\mathbf{0} & \mathbf{w}_{2}^{(g, k)} & \cdots & \mathbf{0} \\
\vdots & \vdots & \ddots & \vdots \\
\mathbf{0} & \mathbf{0} & \cdots & \mathbf{w}_{M}^{(g, k)}
\end{array}\right]
$$

where $\mathbf{0}$ is a $L$-length vector with entries of zeros, $\mathbf{w}_{m}^{(g, k)}$ is the transmitter beamforming vector, which can be optimized with various optimization criteria [13]. In this contribution, specifically, we assume a low-complexity matched-filtering (MF) assisted transmitter beamforming scheme. Furthermore, we assume that the distance between two adjacent array elements of a given antenna array is half of the wavelength of the radio-frequency (RF) carrier. In this case, it can be shown that the weight vector $\mathbf{w}_{m}^{(g, k)}$ achieving the MF-assisted transmitter beamforming is given by

$$
\begin{aligned}
\mathbf{w}_{m}^{(g, k)}= & {\left[1 \quad \exp \left(-j\left[\pi \sin \left(\psi_{m}^{(g, k)}\right)\right]\right) \quad \ldots\right.} \\
& \left.\quad \exp \left(-j\left[(L-1) \pi \sin \left(\psi_{m}^{(g, k)}\right)\right]\right)\right]^{T} \\
m= & 1, \ldots, M ; g=1, \ldots, G ; k=1, \ldots, K
\end{aligned}
$$

where $\psi_{m}^{(g, k)}$ is the DoA in terms of the signals transmitted from the $m$ th antenna array to the $k$ th user in the $g$ th group.

\section{B. Receive Model}

Let the CIR with respect to the $u v$ th subcarrier from the $m$ th BS antenna array to the $k$ th user in the $g$ th group be expressed as

$$
\begin{array}{r}
\overline{\mathbf{h}}_{u v, m}^{(g, k)}=h_{u v, m}^{(g, k)} \cdot\left[1 \quad \exp \left(j\left[\pi \sin \left(\psi_{m}^{(g, k)}\right)\right]\right) \cdots\right. \\
\left.\exp \left(j\left[(L-1) \pi \sin \left(\psi_{m}^{(g, k)}\right)\right]\right)\right]^{T} \\
g=1, \ldots, G ; \quad k=1, \ldots, K ; m=1, \ldots, M ; \\
u=1, \ldots, U ; v=1, \ldots, V
\end{array}
$$

where, again, $\psi_{m}^{(g, k)}$ is the DoA in the context of the signals transmitted from the $m$ th antenna array to the $k$ th user of the $g$ th group, $h_{u v, m}^{(g, k)}$ is the complex-valued fading gain of the $u v$ th subcarrier channel connecting the $m$ th antenna array with the $k$ th user of the $g$ th group.

It can be shown that we have

$$
\overline{\mathbf{h}}_{u v, m}^{(g, k) T} \mathbf{w}_{m}^{(g, k)}=L h_{u v, m}^{(g, k)} .
$$

Therefore, the transmitter beamforming seen in (2) is a MF-based transmitter beamforming scheme, which maximizes the output SNR, when communicating over Gaussian channels without multiuser and inter-channel interference. Let

$$
\overline{\mathbf{h}}_{u v}^{(g, k)}=\left[\overline{\mathbf{h}}_{u v, 1}^{(g, k) T}, \cdots, \overline{\mathbf{h}}_{u v, M}^{(g, k) T}\right]^{T}
$$


which is a $M L$-length vector containing the CIRs of the $M L$ array elements. Then, it can be shown that the inner product between $\overline{\mathbf{h}}_{u v}^{(g, k)}$ and $\mathbf{W}^{(g, j)}$ can be expressed as

$$
\overline{\mathbf{h}}_{u v}^{(g, k) T} \mathbf{W}^{(g, j)}=L \mathbf{h}_{u v}^{(g, k j) T}
$$

where $\mathbf{h}_{u v}^{(g, k j)}$ is a $M$-length CIR vector given by

$$
\mathbf{h}_{u v}^{(g, k j)}=\left[\begin{array}{llll}
h_{u v, 1}^{(g, k j)} & h_{u v, 2}^{(g, k j)} & \cdots & h_{u v, M}^{(g, k j)}
\end{array}\right]^{T}
$$

where $h_{u v, m}^{(g, k j)}$ is given by

$$
h_{u v, m}^{(g, k j)}=h_{u v, m}^{(g, k)} \mathbf{w}_{m}^{(g, k) H} \mathbf{w}_{m}^{(g, j)} / L
$$

which represents the cross-correlation between the $k$ th and $j$ th users' array vectors, both users are in the $g$ th group.

Without loss of any generality, let us focus our attention on the signal received by the first $(k=1)$ user in the first $(g=1)$ group, which we refer to it as the reference user or signal for convenience. Then, the corresponding received signal can be expressed as

$$
r_{u v}(t)=\overline{\mathbf{h}}_{u v}^{T} \times \sum_{g=1}^{G} \mathbf{s}^{(g)}(t)+n_{u v}(t),
$$

where the superscript with the reference user is ignored for convenience. In (11) $\overline{\mathbf{h}}_{u v}^{(g, k)}$ is given by (7), $\mathbf{s}^{(g)}(t)$ is given by (2), while $n_{u v}(t)$ is a complex AWGN noise, which has a mean zero and a variance of $\sigma_{n}^{2}$ per dimension.

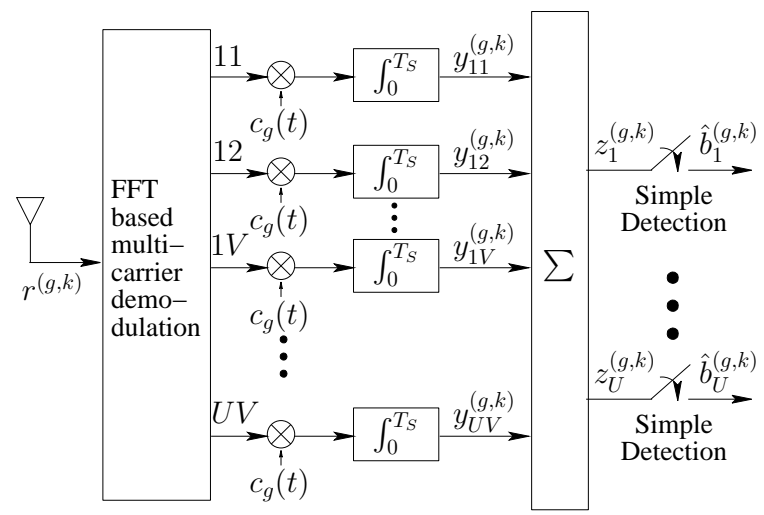

Fig. 2: Receiver schematic block diagram for the $k$ th downlink user in the $g$ th group.

The receiver structure for the $k$ th downlink user in the $g$ th group is shown in Fig. 2. As shown in Fig. 2, after the multicarrier demodulation with the aid of the fast Fourier transform (FFT), the output signals are processed by a bank of $U V$ MFs associated with the spreading code $c_{g}(t)$, which provide $U V$ observations. Finally, for each of the $U$ bits, say $b_{u}^{(g, k)}$, the $V$ observations corresponding to the $V$ subcarriers conveying $b_{u}^{(g, k)}$ is combined, in order to generate the decision variable $z_{u}^{(g, k)}$ for $b_{u}^{(g, k)}$.

According the above discussion and remembering that the $G$ T-domain spreading codes and the different subcarrier signals are orthogonal, it can be shown that the MF's output corresponding to the $u v$ th subcarrier of the reference user can be expressed as

$$
\begin{aligned}
y_{u v} & =\sqrt{\frac{2 P}{V L}} \sum_{k=1}^{K} \overline{\mathbf{h}}_{u v}^{T} \mathbf{W}^{(g, k)} \mathbf{p}_{u v}^{(g, k)} b_{u}^{(g, k)}+n_{u v} \\
& =\sqrt{\frac{2 P L}{V}} \sum_{k=1}^{K} \mathbf{h}_{u v}^{(g, k) T} \mathbf{p}_{u v}^{(g, k)} b_{u}^{(g, k)}+n_{u v}
\end{aligned}
$$

where the elements in $\mathbf{h}_{u v}^{(g, k)}$ are given by (10), while the Gaussian noise sample $n_{u v}$ is given by

$$
n_{u v}=\frac{1}{T_{s}} \int_{0}^{T_{s}} n_{u v}(t) c_{g}(t) \exp \left(-j 2 \pi f_{u v} t\right) d t
$$

which has mean zero and a variance of $\sigma_{n}^{2} / T_{s}$ per dimension.

Finally, the decision variable for $b_{u}^{(g, 1)}$ can be obtained as

$$
z_{u}=\sum_{v=1}^{V} y_{u v}
$$

and $b_{u}^{(g, 1)}$ is decided according to

$$
\hat{b}_{u}^{(g, 1)}=\operatorname{sgn}\left(\Re\left(z_{u}\right)\right), u=1,2, \ldots, U
$$

where $\Re(z)$ represents the real part of $z$.

According to the detection scheme as above-described, the detector consists of a bank of MFs and hence has an extremely lowcomplexity. Furthermore, as seen in (12), the detection is free-from multicarrier interference. There is also no interference from the users in the other groups. However, when the array vectors of the users in the same group are not orthogonal, these users may interfere with each other, yielding the intra-group MUI. In the next section we will show that the intra-group MUI can be fully removed with the aid of the SVD-assisted transmitter preprocessing.

\section{Transmitter Preprocessing Based on Singular VALUE DECOMPOSITION}

In this section we consider the design of the transmitter preprocessing vector $\mathbf{p}_{u v}^{(g, k)}$ seen in (12), so as to achieve simultaneously two objectives: to remove fully the intra-group MUI and to guarantee a transmit diversity order of $n_{t}$ in S-domain. Our design starts with expressing the preprocessing vector $\mathbf{p}_{u v}^{(g, k)}$ as

$$
\mathbf{p}_{u v}^{(g, k)}=\mathbf{P}_{u v}^{(g, k)} \mathbf{d}_{u v}^{(g, k)}
$$

where $\mathbf{P}_{u v}^{(g, k)}$ is $\left(M \times n_{t}\right)$, which is designed to suppress the intragroup MUI, while $\mathbf{d}_{u v}^{(g, k)}$ is a $n_{t}$-length vector used to combine the resultant signals in the S-domain, in order to achieve a diversity order of $n_{t}$ in the S-domain. Let us first consider the design of $\mathbf{P}_{u v}^{(g, k)}$.

\section{A. Design of $\mathbf{P}_{u v}^{(g, k)}$ : Intra-Group MUI suppression}

As shown in (12), the intra-group MUI can be fully removed, if the component preprocessing matrices $\left\{\mathbf{P}_{u v}^{(g, j)}\right\}$ are chosen to satisfy

$$
\mathbf{h}_{u v}^{(g, k) T} \mathbf{P}_{u v}^{(g, j)}=\mathbf{0}_{n_{t}}^{T}, \text { for } k \neq j
$$

where $\mathbf{0}_{n_{t}}$ is a $n_{t}$-length all-zero vector. Therefore, according to [7], the component preprocessing matrix $\mathbf{P}_{u v}^{(g, j)}$ for user $j$ should lie in the null subspace determined by

$$
\begin{array}{r}
\mathbf{H}_{u v}^{(g, j) T}=\left[\mathbf{h}_{u v}^{(g, 1)}, \cdots, \mathbf{h}_{u v}^{(g, j-1)}, \mathbf{h}_{u v}^{(g, j+1)}, \cdots, \mathbf{h}_{u v}^{(g, K)}\right]^{T}, \\
j=1,2, \ldots, K
\end{array}
$$

which is composed by the spatial signatures of the users except user $j$.

Upon carrying out the SVD on $\mathbf{H}_{u v}^{(g, j)}$ [13], we can express it as

$$
\mathbf{H}_{u v}^{(g, j)}=\mathbf{U}_{s, u v}^{(g, j)}\left[\begin{array}{ll}
\mathbf{D}_{u v}^{(g, j)} & \mathbf{0}
\end{array}\right]\left[\begin{array}{c}
\mathbf{V}_{s, u v}^{(g, j) H} \\
\mathbf{V}_{n, u v}^{(g, j) H}
\end{array}\right],
$$

with $\mathbf{U}_{s, u v}^{(g, j)}$ is a $((K-1) \times(K-1))$ orthonormal matrix, $\mathbf{D}_{u v}^{(g, j)}$ is a $((K-1) \times(K-1))$ diagonal matrix containing the non-zero singular values of $\mathbf{H}_{u v}^{(g, j)}, \mathbf{0}$ is a $((K-1) \times(M-K+1))$ all-zero matrix, 
$\mathbf{V}_{s, u v}^{(g, j)}$ is a $(M \times(K-1))$ orthonormal matrix corresponding to the signal subspace determined by the $(K-1)$ interfering users, while $\mathbf{V}_{n, u v}^{(g, j)}$ is a $(M \times(M-K+1))$ orthonormal matrix corresponding to the orthogonal subspace of $\mathbf{H}_{u v}^{(g, j)}$.

Let $n_{t}=M-K+1$. It can be shown that $n_{t}$ represents the transmit diversity order achieved by the MC DS-CDMA system in the S-domain. Hence, $n_{t}$ is referred to as the number of effective transmit antennas. In this case, the component preprocessing matrix $\mathbf{P}_{u v}^{(g, j)}$ can be chosen as

$$
\mathbf{P}_{u v}^{(g, j)}=\mathbf{V}_{n, u v}^{(g, j)}, j=1,2, \ldots, K
$$

Let us now consider the design of the component preprocessing matrix $\mathbf{d}_{u v}^{(g, k)}$ in (16).

\section{B. Design of $\mathbf{d}_{u v}^{(g, k)}$ : Transmit Diversity Combining}

Upon applying the preprocessing matrices $\left\{\mathbf{p}_{u v}^{(g, j)}\right\}$ of (16) associated with the component preprocessing matrices $\left\{\mathbf{P}_{u v}^{(g, j)}\right\}$ of (20) into (12), it can be shown that the intra-group MUI imposed on user 1 is fully removed. Furthermore, the reference user's signal is projected onto a $n_{t}$-dimensional orthogonal subspace. Let

$$
\mathbf{e}_{u v}^{(g, 1)}=\mathbf{P}_{u v}^{(g, 1) T} \mathbf{h}_{u v}^{(g, 1)}
$$

where $\mathbf{e}_{u v}^{(g, 1)}$ can be expressed as

$$
\mathbf{e}_{u v}^{(g, 1)}=\left[\begin{array}{lll}
e_{u v, 1}^{(g, 1)} & \cdots & e_{u v, n_{t}}^{(g, 1)}
\end{array}\right]^{T}
$$

Consequently, we can express the component preprocessing matrix $\mathbf{d}_{u v}^{(g, 1)}$ as

$$
\mathbf{d}_{u v}^{(g, 1)}=\left[d_{u v, 1}^{(g, 1)}, d_{u v, 2}^{(g, 1)}, \ldots, d_{u v, n_{t}}^{(g, 1)}\right]^{T}
$$

and let $d_{u v, i}^{(g, 1)}$ equal to

$$
d_{u v, i}^{(g, 1)}=e_{u v, i}^{(g, 1) *} / \sqrt{E_{u v}^{(g, 1)}}
$$

where $e_{u v, i}^{(g, 1) *}$ is the conjugate of $e_{u v, i}^{(g, 1)}$ and

$$
E_{u v}^{(g, 1)}=\sum_{i=1}^{n_{t}}\left|e_{u v, i}^{(g, 1)}\right|^{2}
$$

is for achieving the constraint on the transmitted power, so that the total transmitted power to a remote user maintains unchanged before and after the preprocessing.

Finally, upon substituting (16) associated with the component preprocessing matrices $\left\{\mathbf{P}_{u v}^{(g, j)}\right\}$ of (20) and $\mathbf{d}_{u v}^{(g, 1)}$ of (23) into (12), it can be shown that the decision variable for $b_{u}^{(g, 1)}, u=1, \ldots, U$ of the reference user can be expressed as

$$
\begin{array}{r}
y_{u v}=\sqrt{\frac{2 P L}{V}} \sqrt{E_{u v}^{(g, k)}} b_{u v}^{(g, k)}+n_{u v}, \\
u=1, \ldots, U ; v=1, \ldots, V
\end{array}
$$

which is free from inter-group interference, multicarrier interference and intra-group MUI.

\section{Discussion}

It is well-known that, in a conventional single-carrier DS-CDMA system [3] using $N_{e}$-chip Walsh-Hadamard (WH) codes as the Tdomain spreading sequences, the maximum number of users supportable is $\mathcal{K}=N_{e}$ without BER degradation in comparison with the corresponding single-user DS-CDMA system. By contrast, the MC
DS-CDMA system considered in this contribution has the following characteristics.

- The diversity order achieved is $V n_{t}=V(M-K+1)$, where $V$ is in the F-domain, while $n_{t}$ is in the S-domain;

- The number of users supportable is $\mathcal{K}=N_{e} K, 1 \leq K \leq M$, where $N_{e}$ is the length of the T-domain spreading sequences, while $K$ is the number of users per group. With the aid of the transmitter preprocessing proposed in Sections III-A and III-B, the $\mathcal{K}=N_{e} K$ number of users can be supported by the MC DS-CDMA system without significant performance degradation in comparison with the single-user MC DS-CDMA system;

- There exists a trade-off between the number of users supported and the diversity order achieved. In the extreme case when the diversity order is $V$ corresponding to $n_{t}=M-K+1=1$, which implies $K=M$, the number of users supportable is as high as $\mathcal{K}=N_{e} M$. If the MC DS-CDMA system supports only $\mathcal{K}=N_{e}$ users implying that each group has only $K=1$ user, then the corresponding diversity order achieved is $V n_{t}=V M$.

Let us now show our simulation results in the next section.

\section{Simulation Result}

In this section the BER performance of the MC DS-CDMA system using multiple BS transmit antenna arrays is investigated, when assuming that each subcarrier signal experiences flat Rayleigh fading. In our simulations we assumed that the F-domain diversity is $V=4$ and that the T-domain spreading sequences were the $N_{e}=32$-length WH codes. The BER performance of the MC DS-CDMA using the proposed transmitter preprocessing is also compared with that of the other MC DS-CDMA systems investigated in the literature as detailed in our forthcoming discourse.

The details about the legends in Figs. 3 and 4 are as follows. For the MC DS-CDMA using the SVD-assisted transmitter preprocessing, the corresponding curves are indicated by ' $\left(M\left(n_{t}\right) \times L\right)$ TP-SVD', where $M$ is the number of transmit antenna arrays, $L$ is the number of array elements of each antenna array and $n_{t}$ represents the number of effective (logical) transmit antennas. In Figs. 3 and 4 the legend ' $(1,1)$ ' indicates the MC DS-CDMA system using no preprocessing, ' $(1, L)$ BF' corresponds to the MC DS-CDMA system using only the steered beamforming, while ' $(M, L)$ SSTS' corresponds to the MC DS-CDMA system using both the steered beamforming and spacetime spreading (STS).

From the results in Figs. 3 and 4, it can be seen that, when assuming orthogonal multicarrier signals and that the subcarrier signals experience flat-fading, a conventional MC DS-CDMA using $N_{e}$-length T-domain spreading sequences is capable of supporting at most $\mathcal{K}=N_{e}$ users without significant BER degradation compared to that of the single-user system. In [5] the MC DS-CDMA system is assumed to employ both T-domain and F-domain, i.e, TF-domain, spreading, in order to extend the maximum number of users supportable by the system. However, as shown in Figs. 3 and 4, when the MC DS-CDMA system using TF-domain spreading supports $\mathcal{K}=2 N_{e}=64$ users instead of $\mathcal{K}=N_{e}=32$ users, the BER performance is significantly degraded. By contrast, when the SVDassisted transmitter preprocessing is employed, as shown in Figs. 3 and 4 , the MC DS-CDMA systems supporting $\mathcal{K}=N_{e}=32$, $\mathcal{K}=2 N_{e}=64$ and $\mathcal{K}=3 N_{e}=96$ users are capable of achieving a similar BER performance.

From the results of Figs. 3 and 4, we can also have the following observations. Firstly, when the MC DS-CDMA system employs only 


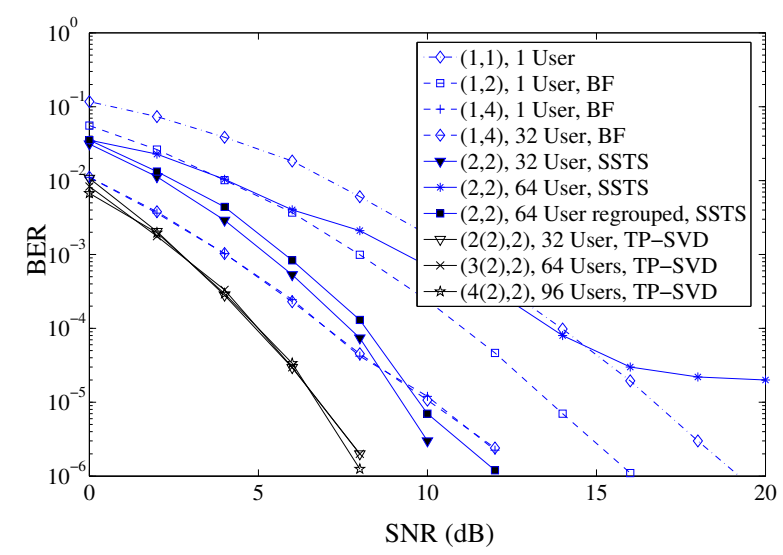

Fig. 3: BER versus SNR performance of the downlink MC DSCDMA system using $V=4$ subcarriers and $N_{e}=32$-chip WH codes based T-domain spreading.

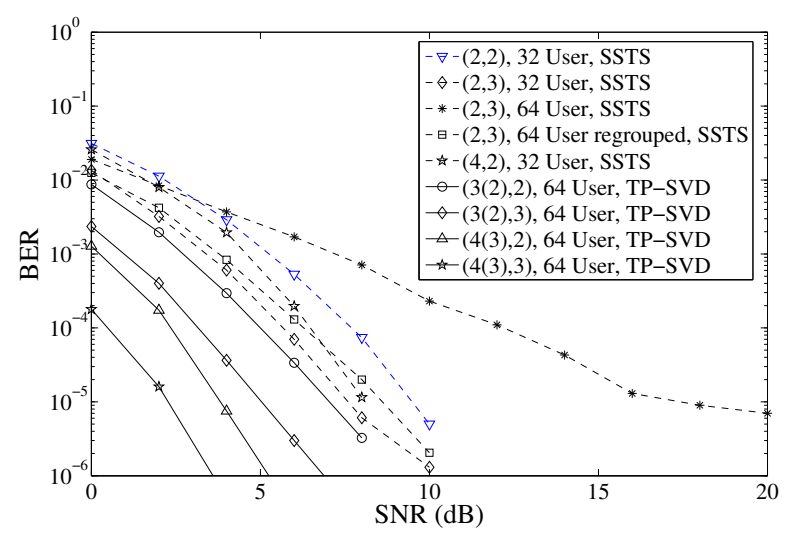

Fig. 4: BER versus SNR performance of the downlink MC DSCDMA systems using $V=4$ subcarriers and $N_{e}=32$-chip WH codes based T-domain spreading.

single transmit antenna array $(M=1)$ with single array element $(L=1)$, the BER performance is the worst. Secondly, when the MC DS-CDMA system employs $M=1 \mathrm{BS}$ antenna array with $L>1$ array elements, the received SNR is significantly improved and the corresponding curves are shifted to the left-hand side of that corresponding to the case of $L=1$. Thirdly, when the MC DS-CDMA system employs $M>1$ BS antenna arrays and each antenna array has $L>1$ array elements, and when the steered transmitter beamforming and STS (SSTS) are applied, the MC DSCDMA system is capable of achieving a transmit diversity order of $M$, in addition to the SNR enhancement due to the antenna arrays [5]. Furthermore, as can be seen in Figs. 3 and 4, when the MC DS-CDMA BS transmitter employs our SVD-assisted transmitter preprocessing indicated by 'TP-SVD', the BER performance is further improved, in comparison with the system using SSTS. In comparison with the SSTS scheme, which does not depend on the knowledge about the downlink CIRs, except the DoAs for the steered beamforming, the SVD-assisted transmitter preprocessing requires the knowledge about the downlink CIRs. However, the SSTS-assisted transmitting scheme demands a relatively higher complexity detection scheme, such as multiuser detection (MUD), than the SVD-assisted transmitter preprocessing, which requires only the low-complexity MF-assisted detection.

\section{CONCLUSION}

In this contribution we have proposed and investigated a SVDassisted transmitter preprocessing scheme for the MC DS-CDMA system using multiple BS transmit antenna arrays, where each antenna array employs multiple array elements. The SVD-assisted transmitter preprocessing has been designed, in order to support possibly a high number of downlink users, while simultaneously to achieve the highest possible transmit diversity gain. Our study shows that, for a SVD-assisted MC DS-CDMA system using $M$ transmit antenna arrays and length- $N_{e}$ T-domain spreading sequences, the number of users supportable can be as high as $M N_{e}$ with the aid of both the T-domain and S-domain multiuser multiplexing. Our simulation results show that the SVD-assisted MC DS-CDMA system is capable of supporting such a high number of users with near singleuser BER performance achieved by a corresponding MC DS-CDMA system supporting only one downlink user.

\section{REFERENCES}

[1] L. L. Yang and L. Hanzo, "Multicarrier DS-CDMA: A multiple-access scheme for ubiquitous broadband wireless communications," IEEE Communications Magazine, vol. 41, no. 10, pp. 116-124, October 2003.

[2] L.-L. Yang and L. Hanzo, "Performance of generalized multicarrier DS-CDMA over Nakagami- $m$ fading channels," IEEE Transactions on Communications, vol. 50, pp. 956 - 966, June 2002.

[3] L.Hanzo, L.-L. Yang, E.-L. Kuan, and K.Yen, Single- and Multi-Carrier DS-CDMA Multi-User Detection, Space-Time Spreading, Synchronisation and Standards. Chichester, UK: John Wiley and Sons, Ltd, 2003.

[4] B. Hu, L.-L. Yang, and L. Hanzo, "Performance of the smart antenna aided multicarrier DS-CDMA uplink," in IEEE 60th Vehicular Technology Conference. IEEE, September 2004, pp. 191 - 195.

[5] B. Hu, L.-L. Yang, and L.Hanzo, "Performance of the smart antenna aided generalized multicarrier DS-CDMA downlink using both timedomain spreading and steered space-time spreading," IEEE 62nd Vehicular Technology Conference,, vol. 1, no. 28-25, pp. 458-462, Sept. 2005.

[6] S. Verdu, Multiuser Detection. Cambridge University Press, 1998.

[7] L.-U. Choi and R. Murch, "A transmit preprocessing technique for multiuser MIMO systems using a decomposition approach," IEEE Transactions on Wireless Communications, vol. 3, no. 1, pp. 20 - 24, 2004.

[8] R. L.-U. Choi and R. D. Murch, "New transmit schemes and simplified receivers for MIMO wireless communication systems," IEEE Transactions on Wireless Communications, vol. 2, no. 6, pp. 1217-1230, November 2003.

[9] R. L.-U. Choi, M.T.Ivrlac, R. D. Murch, and W. Utschick, "On strategies of multiuser MIMO transmit signal processing," IEEE Transactions on Wireless Communications, vol. 3, no. 6, pp. 1936-1941, November 2004.

[10] R. L.-U. Choi and R. D. Murch, "A pre-BLAST-DFE technique for the downlink of frequency-selective fading MIMO channels," IEEE Transactions on Communications, vol. 52, no. 5, pp. 737-743, May 2004.

[11] _ - "A transmit MIMO scheme with frequency domain preequalization for wireless frequency selective channels," IEEE Transactions on Communications, vol. 3, no. 3, pp. 929-938, May 2004.

[12] _ _ "Transmit-preprocessing techniques with simplified receivers for the downlink of MISO TDD-CDMA systems," IEEE Transactions on Vehicular Technology, vol. 53, no. 2, pp. 285-295, March 2004.

[13] S. Haykin, Adaptive Filter Theory, 3rd ed. Upper Saddle River, New Jersey: Prentice Hall, 1996. 